\title{
Space colonization remains the only long-term option for humanity: A reply to Torres
}

\begin{abstract}
Recent discussion of the alleged adverse consequences of space colonization by Phil Torres in this journal is critically assessed. While the concern for suffering risks (s-risks) should be part of any strategic discussion of the cosmic future of humanity, the Hobbesian picture painted by Torres is largely flawed and unpersuasive. Instead, there is a very real risk that the skeptical arguments will be taken too seriously and future human flourishing in space delayed or prevented.
\end{abstract}

Keywords: space colonization - existential risks - futures studies - extraterrestrial intelligence - cosmic optimism

\section{Introduction}

For centuries and millennia, following an ancient Greek ideological obfuscation, people universally and wrongly believed that the magnificent Pyramids of Giza were built by slaves. This belief motivated countless sermons - often self-righteous and Whiggish - about the great engineering projects entailing great human suffering. Modern archeologists discovered, however, not only that workers were paid, free citizens, but the workers' social standing in the contemporary Egyptian society was probably enhanced by their being employed on these sacred projects (e.g., David and David 2002; Smith 2018). We have not heard much of a repentance for heaps of moralistic nonsense of the past. Instead, the focus of the worry about "engineering causing suffering" has just shifted elsewhere.

And a recent favorite of such targets quickly becomes the space colonization. In parallel with the tragic lack of interest in astronautics and space science and technology in general, there is a proliferation of "discourse" mixing skepticism towards space travel and colonization, with either general pessimism, or an anti-enlightenment attitude of downplaying science and technology. Most of such skepticism contains of frivolous and ideologically motivated nonsense which finds the ideas of space colonization and the cosmic future of humanity "racist", "exploitative", "elitist", "diminishing human stature," etc. In this category we might find writings of Arendt [1963] (2007), Williams (2010), Slobodian (2015), Malazita (2017), Kriss (2017), or Klee (2017), among many others. In contrast, the criticism of Torres (2018) is serious, rational, and well-researched, although in the final analysis it is no more definite and persuasive. (Not that there is any real trend in favor of space colonization either in our navel-gazing civilization; as warned by Nunn, Guy, and Bell [2014], we are already in a highly disturbing situation in which the annual cost of obesity in the USA is about 12 times larger (!) than the annual cost of the national space research and exploration programs. That the anti-space sentiment has become completely normative is obvious to such extent that even a self-professed believer in science and 
progress like Steven Pinker calls space colonization a "nonstarter", proposed by "naifs" [Pinker 2018, p. 390]. In this light, all this frantic anti-space opposition looks more and more like flogging of a dead horse.)

Torres argues that human expansion into space will generate a wide variety of distinct posthuman species, many having their own cultural, political, religious, etc. traditions. These descendant species would, according to Torres, engage in perpetual conflicts, resulting in a great suffering. This, allegedly default outcome would constitute a form of "suffering risk". By amply using the views of the $17^{\text {th }}$ century English philosopher Thomas Hobbes, Torres attempts to prove that (p. 84)

\section{[a] closer look at what I have argued are the most probable results of colonizing the "last great frontier" suggests that doing so would yield a state of Hobbesian "warre" in which civilizations wallow in perpetual anxiety-existential anxiety - when they aren't actively engaged in confrontations with their neighbors.}

The least important are several factual mistakes and mispresentations in Torres's account. It is at the very least doubtful whether "individuals like Vasili Arkhipov and Stanislav Petrov more or less singlehandedly averted nuclear holocausts" (p. 82), since neither we could be certain about that, nor they themselves put forward any such claims. The number of human lives lost under the astronomical waste argument is most probably much greater still than the conservative assumption of Bostrom (2003), likely on the order of $10^{46}$ (Ćirković 2004) rather than $10^{38}$ (p. 75). The expansion of the universe does not influence space colonization (p. 79), except in an indirect - and actually favorable to the main thrust of Torres's argument! - way that the accelerated expansion could cause a loss of causal contact between intergalactic colonies. Pathogens which only harm single species are actually a small minority contrary to Torres's assertion (p. 81), and for rather obvious reasons that their virulence decreases the accessible ecological niche; among major pathogens which influenced human history, only smallpox was such an ultra-specialized pathogen. In contrast, many are generalist pathogens, some of which are so flexible that could cause disease in plants as well as animals (e.g., Alberts et al. 2002)! Finally, whether our quantum vacuum is in a stable or a metastable energy state is still the stuff of pure speculation, lacking the definite ultraviolet completion of quantum field theory, such as superstring or M-theory - and it is therefore inappropriate, irresponsible and misleading to assert that "the universe appears to be in a 'metastable' energy state" (p. 81). These are not decisive criticisms, but their misleading nature set the scene for the main thrust of the present argument.

Here, I aim to defend the following theses:

1. Torres's "Hobbesian" view of postbiological evolution is deeply flawed, to the point of inconsistency.

2. The skeptical (about space colonization) argument relies on conflation of several key concepts. Notably:

a. conflation of reasonable and unreasonable future-oriented speculation;

b. conflation of different kinds of colonization of the universe;

c. conflation of "microevolutionary" and "macroevolutionary" implications of the orthogonality thesis of Bostrom.

3. Torres's representation of the logistics of interstellar warfare is highly unlikely at best.

4. If we were to accept the gist of Torres's argument, it would imply moral justification for instituting a constrained, rigid, and unavoidably repressive control of humanity's future here on Earth as well. 
5. The skeptical argument fails to take into account the very real possibility of extraterrestrial life. In the following sections $(2-6)$ I shall consider each of these theses in some detail, before summarizing the failure of skeptical arguments in the concluding section.

\section{What is the meaning of "post" in "postbiological"?}

Torres uses the terms such as "postbiological evolution" or "posthumans", but those concepts do not play their crucial and intrinsic roles in his argument. Therefore, the implied concept of postbiological evolution is inconsistent.

The Hobbesian description of conflict is, obviously, applicable to motivations and drives of biological humans. If the same motivations and drives originating in biological evolution are simply extended into the postbiological realm - what exactly does the "post" stand for? It is not an accidental by-product of postbiological evolution that the biological imperatives are suppressed; it is its very essence. In a schematic way proposed in Ćirković (2008), we need to make an additional step symbolically represented as the analogy:

biological evolution $\rightarrow$ postbiological evolution

necessarily implies

sociobiology $\rightarrow$ "post-sociobiology".

Now, even without knowing what exactly the "post-sociobiology" toolkit will include, we can be reasonably certain that straightforward social consequences of biological evolution, such as aggression, territorial imperative, favoritism toward one's own kin, etc. will be transcended. The origin of warfare and even more broadly, aggression - is obviously of evolutionary origin, grounded in natural selection, as has been known for at least half a century, since the ground-breaking work of Tinbergen (1968). ${ }^{1}$ Often cited and misused "expanding and filling the ecological niches" (e.g., Hanson 1998) are not the intrinsic property of life or intelligence - they are just consequences of the predominant evolutionary mechanism, i.e. natural selection. It is not only logically possible to imagine a situation in which some other mechanism of evolutionary change, like the Lamarckian inheritance or genetic drift, could dominate and prompt different types of behaviour; it is in fact incumbent on any discussion of postbiological evolution to argue that this baggage of natural selection will be left behind. Any postbiological civilization is quite unlikely to retain anything like the genetic lottery when the creation of new generations is concerned. In addition, the easiness of producing and retaining copies of postbiological organisms in the digital substrate are likely to dramatically change the meaning of terms such as "maturation", "adulthood", "parenthood", "kin", etc.

To quote an example used in Ćirković (2008):

Let us, for the sake of elaborated example, consider the society of uploaded minds living in virtual cities of Greg Egan's Diaspora... - apart from some very general energy requirements, making copies of one's mind and even sending some or all of them to intergalactic trips (with subsequent merging of willing copies) is

\footnotetext{
${ }^{1}$ See also Tiger and Fox (1971); Wilson (1978).
} 
cheap and uninfluenced by any biological imperative whatsoever; the galaxy is simply large and they are expanding freely, in many different ways with no clear hierarchy of approaches. There is no genetic heritage to be passed on, no truly threatening environment to exert selection pressure, no necessity to retain biologically determined sexual characters, no biotic competition, no kin selection, no pressure on (digital) ecological boundaries, no minimal viable populations.

(The example used is the upload society described in great detail in the brilliant SF novel Diaspora by Greg Egan [1997].) Therefore, it is not reasonable to expect that the Hobbesian picture of conflict will remain viable any more than the theory of embryonic development from a homunculus, predominant in Hobbes's time, is any more viable. On the contrary, there are reasons to believe that postbiological civilizations will be much more peaceful and stable than the biological ones. ${ }^{2}$ While it is certainly true that there are reasons for conflict other than the inherited evolutionary dispositions in both biological and postbiological societies, like divergent goals and ideologies, two things need to be emphasized. First, we need to be sure that these are indeed reasons, and not only justifications, while keeping in mind that conflict does not entail aggression. Second, the onus of proof lies on those who, like Torres, imply that such non-evolutionary sources of aggression and war are dominant in both biological and postbiological epochs.

\section{Conflations, far and wide}

Torres's arguments often rely on conflation of what can be dubbed "reasonable" and "unreasonable" speculation. For example, to assume that (post)humans will one day colonize and perhaps terraform Mars is a reasonable speculation; to assume that (post)humans will one day develop "weapons that could destroy entire galaxies" (p. 82) is an unreasonable speculation. There are no indications whatsoever that the latter is physically possible. For instance, the binding energy of the stellar subsystem of the Milky Way is about $-6 \times 10^{50} \mathrm{~J}$; if "destruction of the Galaxy" means unbinding all its stars, this would imply the hyperweapon capable of releasing at least that much energy, equivalent to completely converting more than 3,000 Solar-mass stars into usable energy (as per $E=m c^{2}$ ). ${ }^{3}$ This is significantly more than the output of the most energetic known natural processes, including supernovae, gamma-ray bursts, and the most violent QSOs; even more importantly, we cannot envision - if our current astrophysics is correct and there is no bulk antimatter anywhere in the universe - the kind of energy-releasing process necessary for this task. Even if our distant posthuman descendants manage to discover and control such a process, the timescale for such a destruction would of necessity be measured in multiples of $10^{7}$ or $10^{8}$ years, which sounds a bit unreasonable in the tactical sense. And even if a military operation spanning $100,000,000$ years or so could be palatable to a hypothetical future immortals, its efficiency would be doubtful, since not only would the adversary have enough time to prepare, but the unbinding might not automatically mean the destruction of adversary's material and industrial capacities. (And similar objections could be posed to other construals of "destruction", e.g., attempting to collapse all baryonic matter into the central supermassive black hole.)

\footnotetext{
${ }^{2}$ On a related, although significantly more involved note, this is related to another important issue in futures studies, namely the total and abject failure of inductivist reasoning when applied to transformative technologies in general, and postbiological evolution in particular (Walker and Ćirković, in preparation).

${ }^{3}$ In fact, since I have neglected dark matter here, the realistic estimate would be about an order of magnitude higher.
} 
And even if it is possible in some contrived philosophical sense of the "fullness of time" and not in the next billion years or so, there is no need to worry about it, since there are literally thousands of more probable catastrophic scenarios which could occur naturally and which bear no connection to the issue of space colonization. For example, there is a non-vanishing probability that Earth (or the Solar System generally) is on the collision course with an old - and hence hard to detect - neutron star or a black hole. Although highly improbable, such a scenario is still arguably better grounded in our scientific understanding that any "galaxy-destroying" hyperweapon. And it is exactly colonization of space which is the only way of mitigating such cataclysmic collision. The point of this example is, of course, not to make the reader worry about the possibility of such collision; instead, the point is that in futures studies, if we once "cross the Rubicon" by envisioning scenarios of very small probabilities, it is quite easy to reach the conclusions quite opposite to the ones of Torres. For instance, could we not envision a device which will emit "rays of peace", pacifying any sentient being within its radius? ${ }^{4}$ Well, the idea is funny but it is not funnier than the idea of galaxy-destroying hyperweapon. ${ }^{5}$

The necessity of comparing very small probabilities has been recognized in serious risk analysis long time ago. Thus, the "asteroid test" has been instituted: an anthropogenic risk is acceptable as long as it is smaller than the largest natural risk unavoidable at present time (e.g., Calogero 2000; Kent 2004), which has traditionally thought to be the risk of a large asteroid colliding with Earth and causing human extinction. The corresponding probability density has been traditionally put at about $10^{-8}$ per year. ${ }^{6}$ This tells us two things: (i) that we should sleep soundly as far as the "vacuum phase transition" and other ultra-low probability threats are concerned; and (ii) that we can survive on Earth only a minuscule fraction of its remaining habitable time, due to natural cataclysms, if we do not develop massive and extensive space technology. Building of extensive refuges, stockpiling food supplies, protecting data and DNA banks, and similar far-reaching intentional measures could effectively prolong the lifetime of humanity on Earth (e.g., Baum, Denkenberger, and Haqq-Misra 2015; Baum et al. 2015) against both natural and anthropogenic catastrophes. Many of those measures could, however, be performed more efficiently in space than on Earth (e.g., Guzman, Hein, and Welch 2017) and, ironically, some of the technologies involved could as easily be applied in colonization of the Solar System. Above all this is the overarching concern that the terrestrial biosphere is in any case a single system - and uniqueness is always more fragile than multiplicity.

Which leads us to further examples of conflation. Torres fails to clearly distinguish between different kinds of space colonization which correspond to astrophysical distribution of resources. Colonization of our Solar System is one such kind, interstellar colonization within the disk of the Milky Way another, and

\footnotetext{
${ }^{4}$ Cf. Strugatsky and Strugatsky (1971).

${ }^{5}$ The same applies to the speculation of Sandberg et al. (2016) about artificial production of black holes cited by Torres. It is conceived as a domain of extremely advanced Galactic civilizations which have become so scientifically and technologically advanced exactly through space colonization and its benefits. And black holes, parenthetically, are not inherently riskier than stars, asteroids, or even cars, railways or AC plugs at the corresponding level of technological and cultural sophistication.

${ }^{6}$ See Binzel (2000). It is strictly speaking wrong, since the probability density of largest supervolcanic eruptions is for about a factor of two larger for most of the severity spectrum (Mason, Pyle, and Oppenheimer 2004), but it is still useful as a benchmark. The fact that we have identified no asteroids on the collision course so far in spite of the dramatic improvement in observational techniques just underscores this point. See Baum (2018) for a dissenting view on the "asteroid test", however: the probability density of extinction is poorly defined, since human consequences of a large impact has not been studied sufficiently so far.
} 
the intergalactic colonization suggested by Armstrong and Sandberg yet a third one. Neither motivations, not technologies, nor timescales, nor cultural consequences of these kinds are the same, and it is highly misleading to treat them on the same footing. In particular, latency delays of the order of minutes and hours (the Solar System colonization) are rather trivial, those on the order of years or decades (interstellar colonization in Sun's Galactic vicinity) are comparable to those of ancient empires, esp. when human/posthuman life extension is taken into account. Only larger latencies, on the order of millennia (Kardashev Type 3 Galactic civilization) or millions of years or more (intergalactic colonization) are those which imply at least some of the consequences Torres ascribes to all kinds of space colonization.

In the sense that colonization of the Solar System is a necessary precondition for almost any other colonization-related endeavor, we would expect that arguments claiming to overturn the standard way of thinking apply in the strongest to this kind of colonization. However, it is exactly the opposite in Torres's account. By far the most problematic aspects of space colonization apply to the nebulous distant future of galactic colonization; the suggestion that establishing a human colony on Mars would lead to speciation and deadly conflict - any more than what will anyway happen on Earth (see section 5 below) - is unsupported, counterintuitive, and, frankly, naïve.

An especially insidious form of misleading conflation is seen in the following passage (p. 83):

There could also be massive simulations running on exoplanets that have been converted into computronium in which billions of sentient simulants suffer immense agony. Given the huge number of future beings who could exist if we do colonize space, it stands to reason that someone somewhere would run such simulations (perhaps from within a simulation), create new biospheres in which wild animals are subject to Darwinian misery, and so on.

Clearly, the account is biased, since it does not tell us anything about the upside of such simulations, which would counterbalance the negative value created even on the most suffering-averse moral theories. And if humanity refrains from space colonization and inevitably goes extinct - due to either local Hobbesian warfare or a natural cataclysm - wild animals will (surprise, surprise!) continue to be subject to "Darwinian misery" on Earth and on infinite number of other habitable planets in the universe.

Perhaps the least importantly, Torres perhaps inadvertently conflates the two interpretations of the "orthogonality thesis" of Bostrom $(2012,2014)$. Orthogonality thesis as originally conceived applies to the possible evolutionary pathways of a superintelligent Al, or a posthuman species, or perhaps an extraterrestrial intelligent species. Its generalization to a large set of such entities at any particular epoch in time is not warranted, since the evolution of such entities is likely to be strongly non-ergodic (to fill just a small region of the overall parameter space). It is easy to understand why this is so: while the orthogonality thesis allows for a stock example of paperclip maximizer, as well as, for example, spaceship maximizer, it certainly does not tell us that the two are on the same footing in a wider scheme of things. Obviously, the spaceship maximizer would have made a short work of the paperclip maximizer in any realistic conflict, violent or not, if they start from the same initial conditions and distribution of resources. Therefore, it is far likelier that the spaceship maximizer would be able to leave progeny (in sufficiently broad sense of the term) and fill the universe with its spaceship-maximizing values, rather than the paperclip-maximizing values. Therefore, it is naïve to conclude that the validity of the orthogonality thesis suggests that the unconstrained future evolution will bring about equipartition of 
the available design space and that all kinds of posthuman species could be encountered in equal measure. Quite the contrary, it is likely that nonsensical evolutionary trajectories, like those leading to the paperclip maximizing, will be driven to zero relative frequency. Thus, the original, microevolutionary version of the orthogonality thesis does not entail the wider, macroevolutionary version. And we need macroevolutionary insights in order to assess the merits and demerits of our future space colonization efforts.

\section{Interstellar warfare: fantasy and real fantasy}

Even if we accept - in spirit, if not in words - Clarke's Third Law ("any sufficiently advanced technology is indistinguishable from magic"; Clarke 1999), the inverse does not hold: there are infinitely many conceivable "magical" effects which could not be realized with any advanced technology under the known laws of physics. As stated above, it is doubtful, to say at least, that there ever will be a "galaxy destroying weapon", irrespectively of how much time and effort is expended. The influence of science fictional discourse, while in general beneficial for futures studies, becomes at this point perhaps too strong (especially in a rather naïve, Star Wars- or Starship Troopers-like manner). The discussion might be finished here, since it seems unreasonable to engage in such extreme speculation; so, a few comments in the rest of this section should be taken with reservations.

Although there have not been serious strategic studies of the topic, there are many indications that the "interstellar warfare" is an oxymoron. Insofar as there are no topological shortcuts in forms of traversable wormholes (and even if there are some, but with fixed points of entrance and egress), there is a large delay and logistic nightmare in sending any military expeditions across interstellar distances. Except in the case of huge technological imbalance - which is possible in contact between (post)human and some extraterrestrial civilization, but highly unlikely if not impossible between any two (post)human factions - the defense of planetary systems would have overwhelming advantage, measured by orders of magnitude in both reconnaissance, logistics, and capacity for tactical concentration of forces. Above all, the defenders could easily destroy any non-stellar resources in the defended system if their situation becomes desperate enough, so that "piracy and plunder" would simply not be viable options in the interstellar case. And, if the desired resources consist of uplifted stellar matter, there would always be billions of undefended stars in the Galaxy. (This would be valid even if the efficiency of colonization is 99\%. In the unlikely scenario that all stars in the Galaxy are colonized and defended, which is perhaps in itself incompatible with the Torres's argument, since the constant destructive warfare will likely impede or arrest the colonization efforts, the required timescale is so long that it will perhaps make more economic and strategic sense to go after undefended resources in other Local Group galaxies.)

In brief, plunder is hardly viable as a motivation for interstellar warfare. A reasonable conclusion that interstellar travel will always be expensive and difficult, coupled with the defensive advantages and the total abundance of undefended cosmic resources elsewhere, makes this motivation of "Machiavellian actors" largely irrelevant. "Tuckerian actors" have been dealt with in the section 2 above. Finally, there remains the option of warfare for the sake of spreading particular "bad memes" (Zubrin 1999). If this kind of motivation requires the same expenditure of time and resources required for the interstellar travel (not to mention intergalactic travel, which is an entirely different order of magnitude problem, 
with additional difficulties, like the impossibility of gathering any fuel or resources en route), it is reasonable to conclude that such instances would be rare.

Of course, it is impossible to eliminate the possibility that particularly virulent political or religious movements will emerge in the distant future, leading to an overwhelming motivation for spreading The Word by fire and sword even in the face of unfavorable strategic odds. This seems improbable, however, for at least two reasons. (i) The relevant "bad memes" will certainly be easier to spread by other means, notably modulated radiation and inscribed matter packages, in forms of self-reproducing software or other virulent forms, which might be violent in a generalized sense, but still would not entail any of the drastic scenarios of destruction invoked by Torres. Insofar human history is any guide, while religions are stronger in the contemporary world than they have been for the last couple of centuries, this is manifested by televangelists rather than crusades. ${ }^{7}$ (ii) The immunization against such bad memes is quite likely to improve by many orders of magnitude, just as new computer viruses always provoke stronger and more efficient immunization response. In this sense, the conclusions of Pinker (2011) seem fully vindicated; and they are certainly more appropriate for the interstellar case.

\section{A moral case for totalitarianismpreventing diversity?}

Perhaps the darkest side of skeptical arguments is the insistence that the biological and cultural diversity implied by space colonization is dangerous in itself. First, as discussed above - and acknowledged in most discussions of postbiological evolution - there is no sharp boundary between (post)biological morphological and cultural diversities. Second, there is no sharp boundary between the terrestrial and the extraterrestrial realm. It could be argued that, for instance, Earth's orbit or even the Moon are essential parts of the terrestrial realm; this could apply to O'Neill habitats in Earth's orbit or in the stable Lagrangian points of the Sun-Earth system.

Torres's argument here might be simplified as:

1. Diversity is a necessary consequence of space colonization.

2. Diversity inescapably leads to war and suffering.

3. War and suffering are clearly undesirable.

Hence,

\section{Space colonization is clearly undesirable.}

So far, I have concentrated on the suspicious aspects of premise 2. The premise 1 seems rather indisputable. However, why should one not consider the following argument instead:

\footnotetext{
${ }^{7}$ Even jihadist terrorism is more an epiphenomenon of the spread of bad memes via madrasas and internet sites, than a manifestation of an expansionist, proselytizing faith capable of motivating huge and expensive wars of conquest (e.g., Ranstorp 2009). The amount of human suffering created by terrorism is, with full respect towards its victims, negligible in relative terms in comparison to the religious wars and massacres of previous centuries, or even to the world wars created by expansionist ideological memes such as nationalism, fascism, and communism.
} 
1.' Diversity is a necessary consequence of future postbiological evolution and secular increase of freedom on Earth.

2.' Diversity inescapably leads to war and suffering.

3.' War and suffering are clearly undesirable.

Hence,

\section{4.' Postbiological evolution on Earth is clearly undesirable.}

Most treatments of postbiological evolution, including ones of Kurzweil (1999, 2005), Moravec (2000), Smart (2012) and others suggest that postbiological evolution will open new design spaces and lead to unprecedented diversification of future (post)humanity. Traditionally, that has been regarded as a merit rather than a demerit of postbiological evolution. Most of the phenomena alleged to be risky by Torres, such as "cognitive-emotional diversification", "the lack of common ontological ground", "phylogenetic diversity" are not only feasible, but rather highly likely to occur without space colonization either. Even inflicting "eternal punishment" has been conceived and discussed on web fora without any reference to space travel and colonization. All this betrays strong conservatism regarding possible and plausible progress in the social domain: the assumption that improved and more peaceful forms of social organization will not emerge in the future (even cosmologically distant one).

In other words, space colonization is rather peripheral here. In its core, Torres's argument implies an argument against diversity as such and whether such diversity is achieved on Earth or in the near-Earth space or on Mars or in another galaxy is of secondary importance. ${ }^{8}$ And of course, if we once accept that future diversity on Earth will be much higher than anything encountered so far in the course of biological evolution, the size and nature of planetary ecosystem make the conflict much more feasible and hence likelier for rational actors capable of modeling and prediction to achieve their aims - than in the cosmic case. Aggressive actors, if they emerge in the first place (and as per Section 2, I find it unlikely), will have it much easier time in getting to their opponents on Earth, than at distances measured in kiloparsecs. Therefore, diversity and possibility of divergent evolution need to be suppressed (if necessary, by brute force of Hobbesian "Leviathan") here and everywhere.

It is hard to understate the repugnant nature of this conclusion. The whole tradition of liberalism and the Enlightenment is based on assumption that free choice, as a source of all diversity, is of an intrinsic value. In the historical record, the greatest evils inflicted by humans on themselves and their surroundings have not been caused by diversity, but by the exactly opposite tendencies: suppression of diversity and forceful imposition of uniformity. Hence wars of religion, genocides and inquisitions, the Holocaust, Stalinist and Maoist purges, "the killing fields" of Cambodia and other monstrosities of human history. The idea that all this (and immense other) violence is created by diversity, rather than by attempts to impose uniformity, is tantamount to the infamous anti-Semitic meme that only countries

\footnotetext{
${ }^{8}$ Somewhat paradoxically in light of the evolutionary emphasis of Torres's arguments, it seems that the evolutionary history of Homo sapiens shows the advantages of diversity. In particular, this applies to the "out of Africa" theory, and the fact that it seems that rather than killing off Neanderthals, modern humans interbred with them (Wills 2008; Finlayson 2009). This might suggest that even in our cosmic future, the eventual genetic diversity may actually make us stronger, esp. when coupled with cognitive and moral enhancements. I am grateful to an anonymous referee for bringing my attention to this point.
} 
and populations which are already Judenfrei are free of antisemitism. In fact, the suppression of diversity occurred exactly through the immoral violence of Hobbesian "Leviathan" which Torres incomprehensibly and repugnantly - regards as desirable and dubs the consequences "the minor cost of some civilizational freedoms" (p. 79). A very unfortunate consequence is the authoritarian manner in which Torres treats human/posthuman rights. Among many, one rather poignant example is the sentence which starts with "If 'morphological freedom' is granted to martian citizens..." (p. 82) which betrays authoritarian bias - freedoms are not granted, they either are rights or not. If they are rights, then their suppression or revocation is immoral, pure and simple. And it makes Torres's later concern about "the formidable question of what central decision-making body would decide which updates to make" (p. 83) a bit hypocritical. If Pyramids were built by slaves, the undertaking would be immoral, since slavery is inherently immoral; oh, but the Pharaoh could perhaps revoke some freedoms, appropriate for the time, for some "Leviathanic higher purpose", couldn't he?"

Even on skeptical (non-radical!) negative utilitarianism, it is at best unproven that such suppression of diversity would not in itself bring about greater suffering than the diversity itself could bring about. To those of us who regard suppression of personal freedoms - including the essential freedom to diversify - as comparable to, or even worse than, personal extinction, the implications of Torres's argumentation are positively threatening; or at least they would have been, if other parts of the argument were more persuasive. $^{10}$

\section{Extraterrestrial life and utilitarian arguments}

Torres's argument fails to mention the possibility of extraterrestrial life and especially extraterrestrial intelligent life; in this he is quite similar to many other philosophers discussing the future of humanity (e.g., Kahane 2014; Klee 2017). This is important in two different respects. The first is that by relinquishing space colonization, humans obviously leave all available cosmological resources to other intelligent species. (Of course, some of them might be swayed by arguments of extraterrestrial Torresanalogs and relinquish space colonization, but there is no guarantee that all of them will do so, esp. since - as shown above - the arguments are not that persuasive!) This will enable dramatic expansion of an alien colonizing species, which will fill the universe with their own values, entirely different from human/posthuman values. This might or might not be desirable - depending on the character of alien values - but in any case, it would not be particularly appealing from the narrow human perspective. Those averse to s-risks should clearly be against this scenario, since there are no guarantees that aliens would not be more efficient than humans in inflicting suffering onto themselves and others.

The second part of the story is harsher. Ironically, if Torres were right that space colonization would always lead to war between diverging factions, the situation would have been hopeless for humanity,

\footnotetext{
${ }^{9}$ Was King George justified in denying those pesky New England colonists "representation rights"? And would he have been justified if he could somehow predict the suffering inflicted by such increase in diversity not only in the Revolutionary War, but also the war of 1812, etc.?

${ }^{10}$ It is irrelevant for present purposes that a great deal of this argument could apply to Bostrom's singleton as well. Moral aspects of singletons have not been sufficiently studied thus far; it is entirely conceivable that all singletons or all realistic singletons are repugnant in this sense as well. In any case, any conceivable risk of totalitarian usurpation of a future singleton is inherent in the argument that space colonization should be suppressed in order to prevent diversification of future (post)humanity.
} 
since one or another alien faction would have found it both expedient and easy to exterminate or enslave the Earth-bound humanity. By the very logic of Torres's narrative about hyperweapons, the Earth would remain an easy target for any interstellar colonizing faction (and there is no reason to assume that aggressive factions would limit their aggression to their phylogenetic relatives). Thus, Torres's argument is actually self-defeating in the presence of extraterrestrial intelligence - acquiescing to it would directly contribute to the ultimate harm.

Perhaps a skeptic wants to believe (as a kind of anti-agent Moulder, of the X-Files' fame) that extraterrestrial intelligence is non-existent or vanishingly rare? To begin with, it would be strange to bet the long-term future of humanity on such a technical astrobiological issue, on which we can exert no influence whatsoever. Extraterrestrial life either exists or it does not, irrespectively of any amount of our ethical or political hand-wringing. So, lacking specific information for one or the other, we should certainly make strategies for both options. Further, the advances of astrobiology over the last quarter century offer many reasons for cautious belief in the conclusion that life and intelligence are reasonably abundant in astrophysically and astrochemically permissible ecosystems. Some of the arguments to that effect are summarized in Ćirković (2012). ${ }^{11}$ Even if, by some quirk of astrobiological evolution, humanity is the first intelligent species to arise in the Milky Way (as, for instance, per the well-known argument of Carter 1983, 2008), following Torres's advice and relinquishing space colonization will simply ensure that the second, third, or $275^{\text {th }}$ intelligent species to evolve will indeed colonize the Galaxy instead of humans.

If, on the other hand, Torres is wrong and it is possible to colonize the Galaxy in a peaceful and prosperous manner, humanity might survive on Earth in a kind of zoo or preserve, surrounded by friendly and considerate interstellar aliens - but obviously failing to realize its creative potential (which would also count as an existential catastrophe in Bostrom's taxonomy). ${ }^{12}$ There is simply no way out of that quandary, unless one is a creationist who believes that humanity originated by Divine supernatural act and there is exactly zero probability of abiogenesis/noogenesis occurring elsewhere. In general, no naturalistic utilitarian calculus of various scenarios for the future of humanity could be complete if it does not take extraterrestrial intelligence into account.

\section{Conclusions: Space colonization remains the best hope for humanity}

The outline given above shows that the new batch of arguments against space colonization is at best unpersuasive and at worst incoherent. In face of the clear and present danger of existential catastrophes awaiting Earth-locked humanity, it is irresponsible at best to raise the specter of a hypothetical intergalactic war a billion year hence. Expansion has been successful strategy for long-term survival so far among terrestrial species and other taxons, including modern humans; while this might no longer hold in the postbiological regime, we still to reflect more carefully before discarding this default position. Again, it cannot be overemphasized that the study of Torres (2018) is the best of the anticolonization bunch by a long shot; but it is still far from being persuasive. While worrying about possible

\footnotetext{
${ }^{11}$ And the moral argument for our SETI searches has been sketched in Ćirković (2017).

12 Of course, if we relax the anthropocentric concept of the existential catastrophe, this outcome might in fact be highly desirable on various ethical construals; the Galaxy will be peaceful and prosperous due to advanced nonhuman morality. I am grateful to Seth Baum for this important point.
} 
human suffering is legitimate, a more detailed look often shows that the case is - just as with the Pyramid builders - overstated at best. Especially as long as we know much less about what is feasible in the postbiological future than we have known (all along) about the ancient Egyptian civilization.

Note that this is not a defense of maxipok or any other particular consequentialist rule. This is an entirely different and complex topic. Within the framework of this topic, one might ask questions about what fraction of available resources should be allocated for colonizing Moon, Mars, etc., in light of the human failure to seriously colonize Antarctica so far, for example. There is a wide open space for philosophical investigation here. For what it's worth, the present author does not share the view that space colonization needs to be justified within any kind of consequentialist framework. There is a wide spectrum of deontological, virtue-centric, organicist, or rights-centric moral frameworks affirming, at least in principle, the value of space colonization as the best guarantor of human/posthuman survival on cosmological timescales and the spreading of life and reason throughout the universe. Thus, the narrow framing of the issue in terms of maxipok vs. maximin or anything similar is something of a red herring.

Acknowledgements. Useful suggestions and criticisms of two referees for Futures are hereby acknowledged. Detailed comments of Seth Baum, Brian Tomasik, Phil Torres, and Slobodan Perović have been extremely helpful in sharpening the focus of the paper and clarifying key controversial points. I also wish to thank Richard Cathcart, Srdja Janković, Jelena Dimitrijević, Anders Sandberg, Branislav Vukotić, Ksenija Petrović, Nick Bostrom, Slobodan Popović, Karl Schroeder, Aleksandar Obradović, Goran Milovanović, the late Robert Bradbury, and the late Branislav Šimpraga for many pleasant and useful discussions on the topics related to the subject matter of this study. This is an opportunity to thank the KoBSON Consortium of libraries for providing access to the relevant literature. The author acknowledges financial support from the Ministry of Education, Science and Technological Development of the Republic of Serbia through the projects \#ON176021 ("Visible and Invisible Matter in Nearby Galaxies: Theory and Observations") and \#ON179048. ("The Theory and Practice of Science in Society: Multidisciplinary, Educational, and Intergenerational Perspectives").

\section{References}

Alberts, B., et al. 2002, Molecular Biology of the Cell (4 $4^{\text {th }}$ edition, Garland Science, New York).

Arendt, H. [1963] 2007, "The Conquest of Space and the Stature of Man," New Atlantis Fall issue, 43-55 (http://www.thenewatlantis.com/publications/the-conquest-of-space-and-the-stature-of-man, last accessed August 15, 2017).

Armstrong, S. and Sandberg, A. 2013, "Eternity in six hours: Intergalactic spreading of intelligent life and sharpening the Fermi paradox," Acta Astronautica 89, 1-13.

Baum, S. 2018, "Uncertain human consequences in asteroid risk analysis and the global catastrophe threshold," Natural Hazards, in press (doi 10.1007/s11069-018-3419-4). 
Baum, S., Denkenberger, D.C., and Haqq-Misra, J. 2015, "Isolated refuges for surviving global catastrophes," Futures 72, 45-56.

Baum, S., Denkenberger, D. C., Pearce, J. M., Robock, A., and Winkler, R. 2015, "Resilience to global food supply catastrophes," Environment, Systems, and Decisions 35, 301-313.

Binzel, R. P. 2000, "The Torino impact hazard scale," Planetary and Space Science 48, 297-303.

Bostrom, N. 2003, "Astronomical Waste: The Opportunity Cost of Delayed Technological Development," Utilitas 5, 308-314.

Bostrom, N. 2012, "The superintelligent will: Motivation and instrumental rationality in advanced artificial agents," Minds and Machines 22, 71-85.

Bostrom, N. 2014, Superintelligence: Paths, Dangers, Strategies (Oxford University Press, Oxford).

Calogero, F. 2000. Might a laboratory experiment destroy planet Earth? Interdisciplinary Science Reviews 25, 191-202.

Carter, B. 1983, "The anthropic principle and its implications for biological evolution," Philos. Trans. $R$. Soc. London A 310, 347-363.

Carter, B. 2008, “Five- or six-step scenario for evolution?" Int. J. Astrobiol. 7, 177-182.

Ćirković, M.M. 2004, "Forecast for the next eon: Applied cosmology and the long-term fate of intelligent beings," Foundations of Physics 34, 239-261.

Ćirković, M. M. 2008, “Against the Empire," Journal of the British Interplanetary Society 61, 246-254.

Ćirković, M. M. 2012, The Astrobiological Landscape: Philosophical Foundations of the Study of Cosmic Life (Cambridge University Press, Cambridge).

Ćirković, M. M. 2017, "Enhancing a Person, Enhancing a Civilization: A Research Program at the Intersection of Bioethics, Future Studies, and Astrobiology," Cambridge Quarterly of Healthcare Ethics 26, 459-468.

Clarke, A. C. 1999, Profiles of the Future: An Inquiry Into the Limits of the Possible (Gollancz, London).

Cockell, C. S. 2007, Space on Earth: Saving Our World by Seeking Others (Macmillan, New York).

Cockell, C. S. 2008, "An Essay on Extraterrestrial Liberty," Journal of the British Interplanetary Society 61, 255-275.

David, A. R. and David, R. 2002, The Pyramid Builders of Ancient Egypt: A Modern Investigation of Pharaoh's Workforce (Routledge, Abingdon).

Egan, G. 1997, Diaspora (Orion/Millennium, London).

Finlayson, C. 2009, The Humans Who Went Extinct: Why Neanderthals Died out and We Survived (Oxford University Press, Oxford).

Guzman, M., Hein, A. M., and Welch, C. 2017, "Extremely long-duration storage concepts for space," Acta Astronautica 130, 128-136. 
Hanson, R. 1998, "The great Filter - are we almost past it?" (preprint at http://hanson.gmu.edu/greatfilter.html, last accessed September 15, 2017).

Kahane, G. 2014, “Our Cosmic Insignificance,” Nous 48, 745-772.

Kent, A. 2004. A critical look at risk assessments for global catastrophes. Risk Analysis 24, 157-168.

Klee, R. 2017, “Human expunction," International Journal of Astrobiology 16, 379-388.

Kriss, S. 2017, "Think Twice About Escaping Earth to an Exoplanet," The Atlantic March 2017, https://www.theatlantic.com/science/archive/2017/03/space-travel-wont-save-you-fromcapitalism/518853/ (last accessed May 27, 2018).

Kurzweil, R. 1999, The Age of Spiritual Machines: When Computers Exceed Human Intelligence (Viking, New York).

Kurzweil, R. 2005, The Singularity Is Near: When Humans Transcend Biology (Viking, New York).

Malazita, J. W. 2017, “Astrobiology's Cosmopolitics and the Search for an Origin Myth for the Anthropocene," Biological Theory 13, 113-120.

Mason, B.G., Pyle, D.M. and Oppenheimer, C., 2004, "The size and frequency of the largest explosive eruptions on Earth," Bulletin of Volcanology 66, 735-748.

Moravec, H. 2000, Robot: Mere Machine to Transcendent Mind (Oxford University Press, Oxford).

Nunn, A. V. W., Guy, G. W., and Bell, J. D. 2014, "The intelligence paradox; will ET get the metabolic syndrome? Lessons from and for Earth," Nutrition \& Metabolism 11, 34 (13pp).

Pinker, S. 2011, The better angels of our nature: Why violence has declined (Penguin Books, New York).

Pinker, S. 2018, Enlightenment Now: The Case for Reason, Science, Humanism, and Progress (Penguin Books, New York).

Ranstorp, M. 2009, Understanding Violent Radicalisation (Routledge, London).

Slobodian, R. E. 2015, "Selling space colonization and immortality: A psychosocial, anthropological critique of the rush to colonize Mars," Acta Astronautica 113, 89-104.

Smart, J. S. 2012, "The transcension hypothesis: Sufficiently advanced civilizations invariably leave our universe, and implications for METI and SETI," Acta Astronautica 78, 55-68.

Smith, C. B. 2018, How the Great Pyramid Was Built (Smithsonian Institution, Washington).

Strugatsky, A. N. and Strugatsky, B. N. 1971, Inhabited Island (Обитаемый остров, Детская литература, Moscow; in Russian)

Tiger, L. and Fox, R. 1971, The Imperial Animal (Holt, Rinehart \& Winston, New York).

Tinbergen, N. 1968, “On war and peace in animals and man," Science 160, 1411-1418.

Torres, P. 2018, “Space Colonization and Suffering Risks: Reassessing the 'Maxipok Rule,'” Futures 100, 74-85. 
Williams, L. 2010, “Irrational Dreams of Space Colonization," Peace Review 22, 4-8.

Wills, C. 2008, "Evolution theory and the future of humanity," in N. Bostrom and M. M. Ćirković (eds.) Global Catastrophic Risks (Oxford University Press, Oxford), 48-72.

Wilson, E. O. 1978, On Human Nature (Harvard University Press, Cambridge).

Zubrin, R. 1999, Entering Space: Creating a Spacefaring Civilization (Jeremy P. Tarcher/Putnam, New York). 